\title{
Colorectal involvement of follicular lymphoma - findings with narrow band imaging and magnifying endoscopy
}

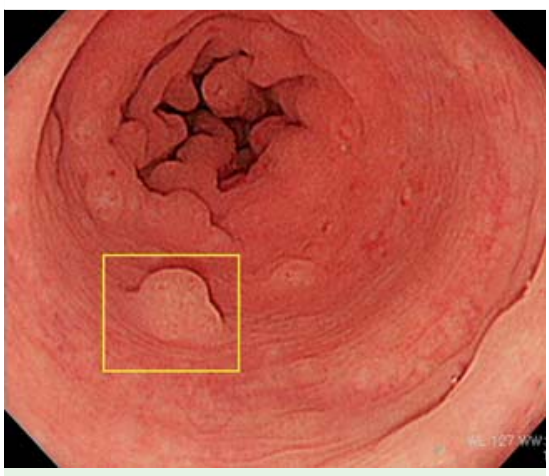

Fig. 1 Total colonoscopy revealed multiple lymphomatous polyposis.

Follicular lymphoma occurs rarely in the gastrointestinal tract. Although prior studies have reported cases of follicular lymphoma in the gastrointestinal tract by using advanced endoscopic imaging studies [1 - 3], observation of colorectal involvement of follicular lymphoma using magnifying endoscopy with narrow band imaging (ME-NBI) has rarely been described.

A 69-year-old woman with follicular lymphoma underwent colonoscopy to investigate mild anemia and bloody stool. Colonoscopy showed multiple lymphomatous polyposis (MLP) through the whole colorectum ( $\bullet$ Fig. 1). ME-NBI highlighted coiled and elongated microvascular patterns on the surface of each lesion, and white opaque spots under the microvessels ( Fig.2). The microscopic examination of samples taken from the lesions showed the follicular structures consisting of medium-sized abnormal lymphoid cells with dense nuclei ( $\bullet$ Fig.3). Immunohistochemical analysis revealed that the neoplastic cells were positive for CD20 and Bcl-2, but negative for CD3, and hence the patient was diagnosed as having colorectal involvement of follicular lymphoma.

We report a rare case of the colorectal involvement of follicular lymphoma, mani-
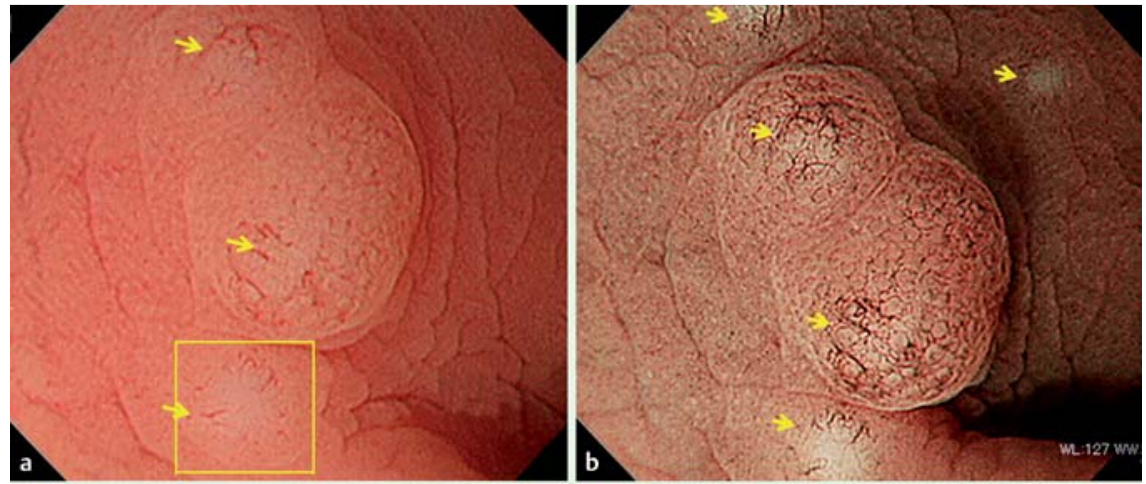

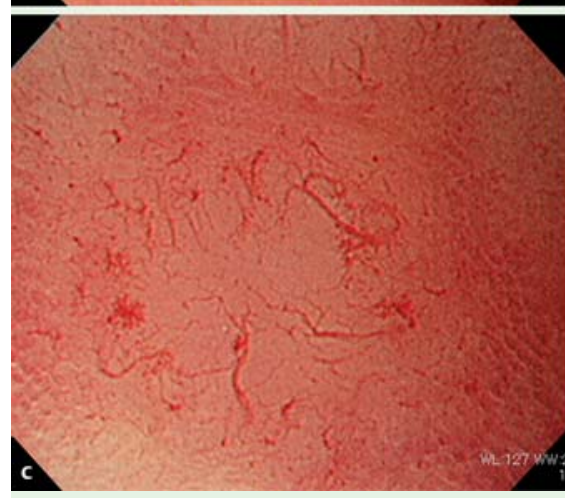

Fig. 2 Magnifying endoscopy of the area indicated by a yellow rectangle in $\$$ Fig. 1: moderate zoom (a, b); full zoom (c, d). a Magnifying endoscopy showed abnormal microvessels on the surface of each lesion and lymphoid follicles (arrows). b Magnifying endoscopy with narrow band imaging highlighted coiled and elongated microvascular patterns on the surface of each

festing as MLP. The endoscopic findings with ME-NBI of colorectal follicular lymphoma have not been clarified. In this case, ME-NBI clearly identified the characteristic coiled and elongated microvascular patterns at the surface of each lesion, and white opaque spots under the microvessels. We believe that ME-NBI is useful for the diagnosis of colorectal follicular lymphoma.

\section{Endoscopy_UCTN_Code_CCL_1AD_2AC}

Competing interests: None

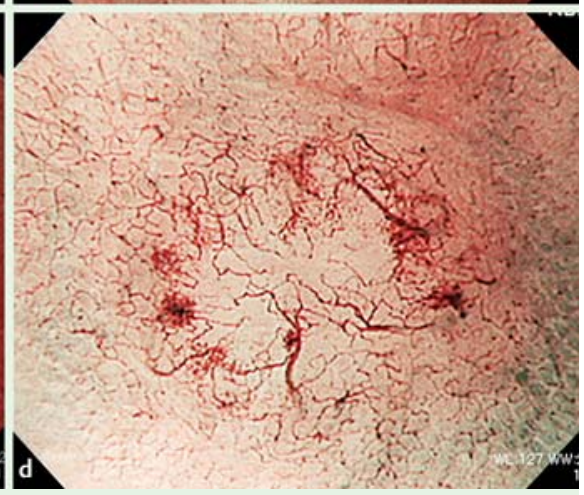

lesion, and white opaque spots (arrows) under the microvessels. c Magnifying endoscopy of the area indicated by the yellow rectangle in - Fig. 2 a. d Magnifying endoscopy with narrow band imaging highlighted coiled and elongated microvascular patterns on the surface of each lesion.

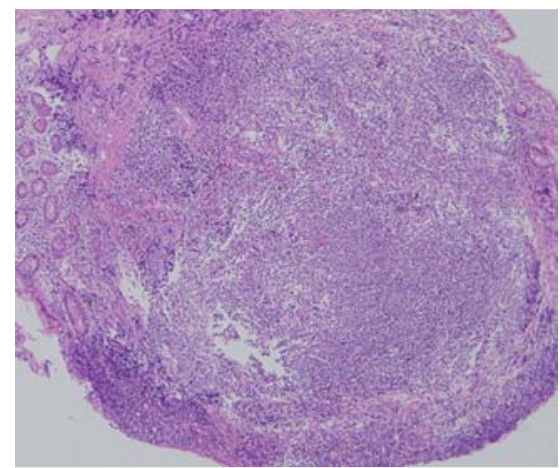

Fig.3 The microscopic examination of samples taken from the lesions showed the follicular structures consisting of medium-sized abnormal lymphoid cells with dense nuclei. 


\section{Norimura' ${ }^{1}$, H. Isomoto ${ }^{2}$}

${ }^{1}$ Department of Gastroenterology and Hepatology, Sasebo Municipal General Hospital, Sasebo, Japan

2 Department of Gastroenterology and Hepatology, Nagasaki University Hospital, Nagasaki, Japan

\section{References}

1 Kuroha $M$, Endo K, Sato $Y$ et al. Magnifying endoscopy findings in follicular lymphoma of the rectum using narrow band imaging. Endoscopy 2011; 43: E346-347

2 Inoue $\mathrm{N}$, Isomoto $\mathrm{H}$, Shikuwa $\mathrm{S}$ et al. Magnifying endoscopic observation of primary follicular lymphoma of the duodenum by using the narrow-band imaging system. Gastrointest Endosc 2009; 69: 158 - 159

3 Norimura $D$, Isomoto $H$, Imaizumi $Y$ et al. Case series of duodenal follicular lymphoma, observed by magnified endoscopy with narrow-band imaging. Gastrointest Endosc 2011; 74: 428-434
Bibliography

DOI http://dx.doi.org/

10.1055/s-0032-1309393

Endoscopy 2013; 45: E296-E297

(c) Georg Thieme Verlag KG

Stuttgart · New York

ISSN 0013-726X
Corresponding author

D. Norimura, MD

Sasebo Municipal General Hospital 9-3 Hirase

Sasebo $857-8511$

Japan

Fax: +81-95-6224641

norir333081@yahoo.co.jp 